\title{
Fault-Tolerant and Scalable Protocols for Replicated Services in Mobile Agent Systems ${ }^{\star}$
}

\author{
JinHo Ahn ${ }^{1}$ and Sung-Gi Min ${ }^{2, \star \star}$ \\ 1 Dept. of Computer Science, Kyonggi University, Republic of Korea, \\ jhahn@kyonggi.ac.kr \\ 2 Dept. of Computer Science \& Engineering, Korea University, Republic of Korea, \\ sgmin@korea.ac.kr
}

\begin{abstract}
To enhance scalability of replicated services a large number of mobile agents attempt to access in mobile agent systems, we present a new strategy to apply an appropriate passive replication protocol to each replicated service according to its execution behavior because deterministic services require weaker constraints to ensure their consistency than nondeterministic ones. For this goal, two passive replication protocols are introduced for non-deterministic services and for deterministic services respectively. They both allow visiting mobile agents to be forwarded to and execute on any node performing a service agent, not necessarily the primary agent. Especially, in case of the protocol for deterministic services, after a backup service agent has received a mobile agent request and obtained the delivery sequence number of the request from the primary service agent, the backup agent, not the primary one, is responsible for processing the request and coordinating with the other replica service agents.
\end{abstract}

\section{Introduction}

Mobile agent paradigm is considered as a promising vehicle for developing distributed computing systems such as grid computing, e-commerce, active networks and embedded systems because it provides a number of advantages such as reduction of network traffic and asynchronous interaction and so on unlike the traditional client server paradigm [1,5,7]. As the mobile agent system is gaining popularity and the number of mobile agent's users rapidly increases, a large number of mobile agents may concurrently be transferred to a node supporting a particular service. In this case, the service agent on the node can be a performance bottleneck and if the agent fails, the execution of all the transferred mobile agents be blocked. In order to solve these problems, the service agent function should be replicated at multiple nodes. This approach may balance the load caused by the mobile agents and even if some service agents crash, continuously allow the other service agents to provide the mobile agents with the

\footnotetext{
* This work was supported by Korea Research Foundation Grant.(KRF-2002-003D00248).

${ }^{\star \star}$ Corresponding author. Tel.:+82-2-3290-3201; fax:+82-2-953-0771.
} 
service. There are two approaches used in distributed systems to potentially be applied for satisfying the goal: active and passive replication [8]. Among the two approaches, the passive replication approach [3, 4] has three desirable features we focus on. First, the approach enables its consistency to be guaranteed even if replicated service agents are performed in a non-deterministic manner. Thus, it can be applied to every replicated service regardless of the execution behavior of the service. Second, it needs lower processing power during failure-free execution than the active replication one. Third, mobile agents has only to use a unicast primitive, not a multicast one because they send service requests only to the primary service agent. But, the traditional passive replication approach may result in some scalability and performance problems when being applied to the mobile agent system as a fault-tolerant technique for replicated services. In other words, to the best of our knowledge, previous works [3, 4] uniformly applied the traditional passive replication approach to each replicated service regardless of whether it is deterministic or non-deterministic. But, in this approach, every mobile agent request should be sent only to the primary service agent, which processes the request and coordinates with the other live replicas and then returns a response of the request to the mobile agent. This special role of the primary is necessarily required to ensure the consistency for non-deterministic services. Moreover, the traditional passive replication approach forces all visiting mobile agents to be transferred to and execute their works in order only on the node running the primary service agent of each domain. These inherent features may cause the extreme load condition to occur on the primary service agent when a large number of mobile agents are forwarded to the service domain and access its resources. Thus, this previous strategy may not achieve high scalability and performance. This paper presents a scalable strategy to apply an appropriate passive replication protocol to each service according to its execution behavior because deterministic services require weaker constraints to ensure their consistency than non-deterministic ones. For this goal, two passive replication protocols are designed in this paper. The first protocol for non-deterministic services is named $P R P N S$ and the second protocol for deterministic services, $P R P D S$. They both allow visiting mobile agents to be forwarded to and execute on any node performing a service agent, not necessarily the primary agent. Especially, in case of the second protocol PRPDS, after a backup service agent has received a mobile agent request and obtained the delivery sequence number of the request from the primary service agent, the backup agent, not the primary one, is responsible for processing the request and coordinating with the other replica service agents. Due to this feature, $P R P D S$ is more lightweight than $P R P N S$ tolerating non-deterministic servers such as multi-threaded servers.

\section{The Proposed Passive Replication Protocols}

In here, we attempt to improve scalability of mobile agent systems by using the appropriate passive replication protocol for each replicated service domain 
according to whether the service is deterministic or non-deterministic. For this purpose, two passive replication protocols $P R P N S$ and $P R P D S$ are introduced.

When mobile agents are concurrently transferred to a non-deterministic replicated service, the consistency is ensured by applying the traditional passive replication protocol. When mobile agent $a_{j}$ attempts to use the service of a non-deterministic replicated service domain $u_{i}, a_{j}$ should be transferred from its current node to the node where the primary service agent of $u_{i}, u_{i}^{\text {prim }}$, executes. Then, the following phases are performed:

(Phase 1). When $u_{i}^{\text {prim }}$ receives a request message from mobile agent $a_{j}, u_{i}^{\text {prim }}$ processes the message. After the execution, it generates (response,psn, next state), where response is the response of the message, psn identifies the processed request message and next_state is the state of $u_{i}^{\text {prim }}$ updated by processing the request.

(Phase 2). $u_{i}^{\text {prim }}$ sends all backup service agents the update message (response, psn, next_state, $j$, reqid) using View Synchronous Multicast(VSCAST) 8 respectively, where $j$ identifies $a_{j}$ and reqid is the send sequence number of the request message. In here, $V S C A S T$ is the multicast communication primitive used to ensure correctness of the passive replication approach. When each backup service agent receives the update message, it updates its state using next_state, maintains (response, $j$, reqid) in its buffer and then sends an acknowledgement message to $u_{i}^{\text {prim }}$. In this case, (response, $j$, reqid) is needed to ensure the exactly once semantics despite $u_{i}^{\text {prim }}$, failure.

(Phase 3). After receiving an acknowledgement message from every live backup service agent, $u_{i}^{\text {prim }}$ sends response to $a_{j}$.

Therefore, this protocol forces all visiting mobile agents to be transferred to and execute their works in order only on the node running the primary service agent of each domain. This behavior may cause the extreme load condition to occur when a large number of mobile agents are forwarded to the service domain to use the non-deterministic service. For example, in figure 1, three mobile agents $a_{t}^{l}, a_{s}^{m}$ and $a_{r}^{n}$ are concurrently transferred to the node node $_{i}^{2}$ where the primary service agent of $u_{i}, u_{i}^{2}$, is running. The mobile agents perform the service of $u_{i}$ in order via only $u_{i}^{2}$ using the above mentioned protocol respectively. Thus, we introduce a passive replication protocol for non-deterministic service, PRPNS, to solve the problem by allowing mobile agents to be forwarded to and execute on each a node performing a service agent, not necessarily the primary agent. The protocol forces each service agent $p$ to forward every request received from mobile agents to the primary service agent. Afterwards, the primary agent performs the phases 1 through 2 previously mentioned to satisfy the consistency condition for non-deterministic service. After receiving an acknowledgement from every normal backup service agent, the primary agent sends the response of the request to the service agent $p$, which forwards it to the corresponding mobile agent. 


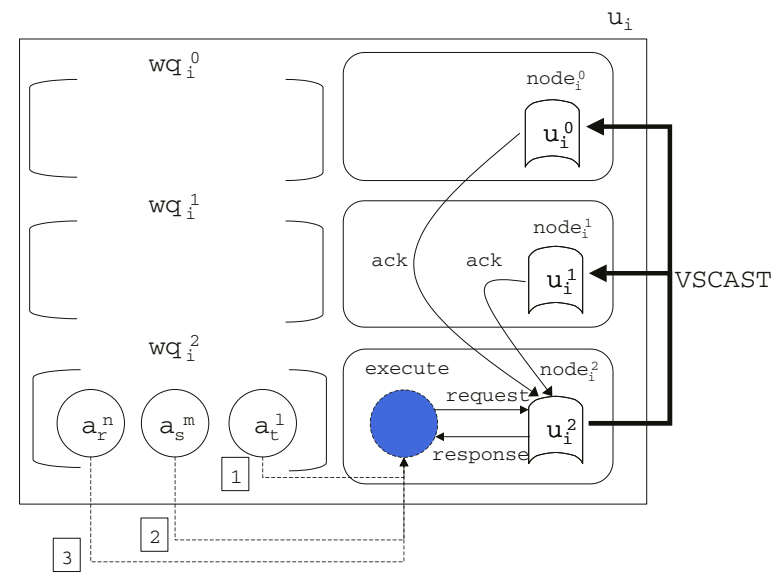

Fig. 1. an execution of three mobile agents accessing a replicated service $u_{i}$ in the traditional passive replication protocol

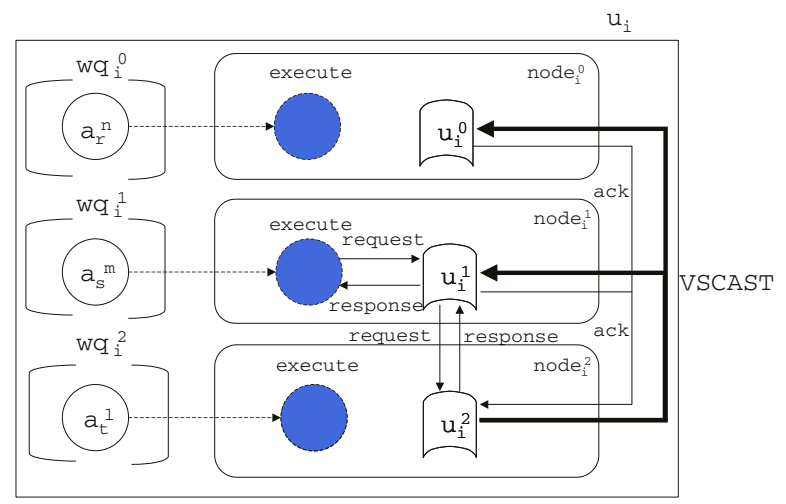

Fig. 2. an execution of three mobile agents accessing a replicated service $u_{i}$ in the protocol PRPNS

Figure 2 illustrates how the protocol PRPNS executes. In this figure, mobile agents $a_{t}^{l}, a_{s}^{m}$ and $a_{r}^{n}$ are transferred to and execute on the nodes node $e_{i}^{2}$, node $_{i}^{1}$ and node $_{i}^{0}$ for accessing service $u_{i}$. Then, the mobile agents perform their tasks via $u_{i}^{2}, u_{i}^{1}$ and $u_{i}^{0}$ respectively. For example, if $u_{i}^{1}$ receives a request from $a_{s}^{m}$, it forwards the request to the primary service agent $u_{i}^{2}$. Afterwards, $u_{i}^{2}$ processes the request and coordinates with the other service agents $u_{i}^{1}$ and $u_{i}^{0}$ using $V S C A S T$. When receiving acknowledgements from the two backup service agents, it sends the response of the request to $u_{i}^{1}$, which forwards the response to $a_{s}^{m}$. From this example, we can see that PRPNS improves scalability of replicated services in case a large number of mobile agents attempt to use a particular service simultaneously. If a mobile agent accesses a service via a backup service agent in this protocol, two more messages are required per request compared 
with the traditional protocol. However, the additional message cost is not significant because each service domain is generally configured on a local area network like Ethernet.

A deterministic replicated service requires weaker constraints to ensure the consistency than a non-deterministic one. In other words, after the primary service agent has determined the processing order of every request from mobile agents, it is not necessary that only the primary agent handles all requests, and coordinates with the other replica service agents like in the protocol PRPNS. With this observation, we attempt to use a lightweight passive replication protocol, PRPDS, for each deterministic service domain. The proposed protocol has the following features.

- Each mobile agent can use a service via the primary service agent or a backup one.

- Only the primary service agent determines the processing order of every request from mobile agents.

- After a backup service agent has received a mobile agent request and obtained the order of the request from the primary service agent, the backup agent, not the primary one, processes the request and coordinates with the other replica service agents including the primary agent.

Due to these desirable features, this protocol enables each visiting mobile agent to be forwarded to and execute on a node running any among replicated service agents in the service domain. If mobile agent $a_{j}$ is transferred to the node where a backup service agent $u_{i}^{\text {backup }}$ executes, PRPDS executes the following phases. Otherwise, the three phases of PRPNS are performed.

(Phase 1). When $u_{i}^{\text {backup }}$ receives a request message from $a_{j}, u_{i}^{\text {backup }}$ asks the primary service agent $u_{i}^{\text {prim }}$ the psn of the request message. In this case, after $u_{i}^{\text {prim }}$ determines the $p s n$ of the message, it notifies $u_{i}^{\text {backup }}$ of the $p s n$. Then, $u_{i}^{\text {prim }}$ processes the request message and saves (response,psn, next_state, $j$, reqid) of the message in its buffer. When receiving the $p s n, u_{i}^{\text {backup }}$ processes the corresponding request and generates (response,psn, next_state) of the request.

(Phase 2). $u_{i}^{\text {backup }}$ sends the other service agents the update message (response, psn, next_state, j, reqid) using $V S C A S T$ respectively. When each service agent except for $u_{i}^{\text {prim }}$ receives the update message, it updates its state using next_state, maintains (response, $j$, reqid) in its buffer and then sends an acknowledgement message to $u_{i}^{b a c k u p}$. If $u_{i}^{\text {prim }}$ receives the update message from $u_{i}^{\text {backup }}$, it just removes the element (response,psn, next_state, j,reqid) for the message from its buffer, saves (response, $j$, reqid) in the buffer and sends an acknowledgement message to $u_{i}^{\text {backup }}$.

(Phase 3). Once $u_{i}^{\text {backup }}$ receives an acknowledgement message from every other live service agent, it sends response to $a_{j}$.

Figure 3 shows an execution of three mobile agents $a_{t}^{l}, a_{s}^{m}$ and $a_{r}^{n}$ attempting to use service $u_{i}$ via $u_{i}^{2}, u_{i}^{1}$ and $u_{i}^{0}$ in the protocol PRPDS. In this figure, only the 


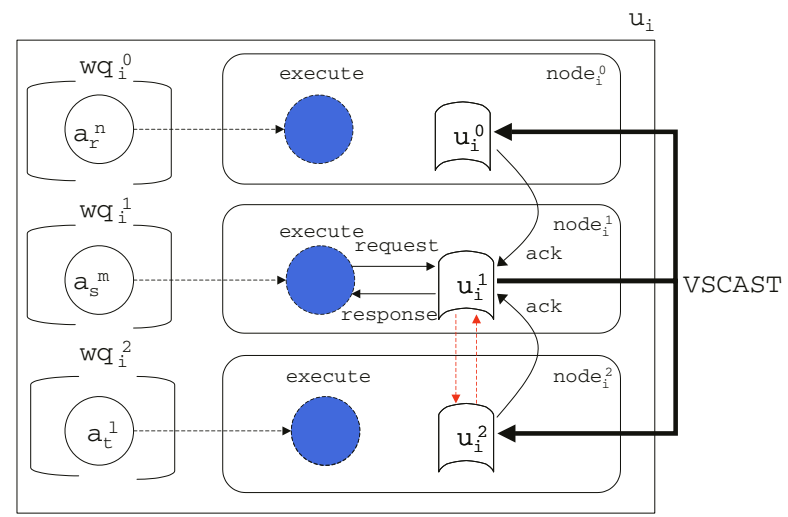

Fig. 3. an execution of three mobile agents accessing a replicated service $u_{i}$ in the protocol PRPDS

procedure is pictured to execute in $P R P D S$ when $a_{s}^{m}$ sends a request to $u_{i}^{1}$. In this case, $u_{i}^{1}$ asks the primary agent $u_{i}^{2}$ the $p s n$ of the request. $u_{i}^{2}$ determines the $p s n$ and sends it to $u_{i}^{1}$. Then, $u_{i}^{2}$ processes the request and saves (response, psn, next_state, $j+1$, reqid) of the request in its buffer. Meanwhile, after obtaining the psn of the request from the primary agent, $u_{i}^{1}$ processes the request and coordinates with the other service agents $u_{i}^{2}$ and $u_{i}^{0}$ using $V S C A S T$ to satisfy the consistency condition for deterministic service. When $u_{i}^{1}$ receives an acknowledgement from every live agent, it sends the response to $a_{s}^{m}$. From this figure, we can see that the protocol $P R P D S$ may significantly improve scalability of replicated services by enabling each visiting mobile agent to use a particular service via any among replicated service agents on the service domain and the request processing and coordination load to be distributed between a set of service agents.

\section{$2.1 \quad$ Recovery}

If service agents fail on a service domain, the proposed two protocols, PRPNS and $P R P D S$, should perform their recovery procedures to satisfy each consistency condition for nondeterministic service or deterministic service despite the failures respectively. Firstly, in case of the crash of the primary service agent $u_{i}^{\text {prim }}$, the two protocols execute both each appropriate recovery procedure in the following cases.

(Case 1). The primary service agent $u_{i}^{\text {prim }}$ fails before finishing the phase 1.

In this case, a new primary service agent $u_{i}^{\text {prim }}$ is elected among all backup service ones. Since no response of each request can be received from $u_{i}^{\text {prim }}$, the request will be resent to $u_{i}^{\text {prim }}$, which has only to perform the phases 1 through 3 .

(Case 2). The primary service agent $u_{i}^{\text {prim }}$ fails after completing the phase 1 , but before sending the response to the corresponding mobile agent. 
Like in case 1 , a new primary agent $u_{i}^{\text {prim }}$ is selected. To ensure linearizability in this case, either all the backup service agents receive the update message, or none of them receive it. $V S C A S T$ is used to satisfy the atomicity condition of linearizability in case of an asynchronous mobile agent system with an unreliable failure detector. If no backup service agent receives the update message, this case is similar to case 1. Otherwise, (response, j, reqid) in the phase 2 is used to ensure the exactly-once semantics. In other words, when the request from the corresponding mobile agent is sent to the new primary service agent $u_{i}^{\text {prim }}$ again, the latter immediately sends the response of the request to the first without handling the request.

(Case 3). The primary service agent $u_{i}^{\text {prim }}$ fails after finishing the phase 3 . Like in case 1 , a new primary service agent $u_{i}^{\text {prim }}$ is selected and identified.

Secondly, when a backup service agent $u_{i}^{\text {backup }}$ crashes, PRPNS and PRPDS perform their recovery procedures as follows. In $P R P N S, u_{i}^{b a c k u p}$ is just removed from its service group. On the other hand, PRPDS executes a corresponding recovery procedure in each following case.

(Case 1). Backup service agent $u_{i}^{b a c k u p}$ fails before asking the primary service agent $u_{i}^{\text {prim }}$ the $p s n$ of each request in the phase 1 .

In this case, $u_{i}^{\text {backup }}$ is just removed from its group. Afterwards, $u_{i}^{\text {backup, }} \mathrm{s}$ failure is detected because of no response of the request from it.

(Case 2). Backup service agent $u_{i}^{\text {backup }}$ fails before finishing the phase 1 . (Case 2.1). The primary $u_{i}^{\text {prim }}$ fails.

The other backup service agents select a new primary service agent, $u_{i}^{\text {prim }}{ }^{\prime}$, among them.

(Case 2.2). The primary $u_{i}^{\text {prim }}$ is alive.

Detecting $u_{i}^{\text {backup }}$ 's crash, $u_{i}^{\text {prim }}$ retrieves every update information, which is form of (response,psn, next_state, $j$,reqid), from its buffer and sends it to the other live backup service agents respectively. In this case, $V S C A S T$ is used to ensure linearizability.

(Case 3). Backup service agent $u_{i}^{\text {backup }}$ fails after sending the update message to the other service agents in the phase 2 , but before sending the response to the corresponding mobile agent.

As $u_{i}^{\text {backup }}$ sent the update message to the other service agents by using $V S C A S T$, the entire consistency is ensured. Therefore, the service group of $u_{i}^{\text {backup }}$ has only to remove $u_{i}^{\text {backup }}$.

(Case 4). Backup service agent $u_{i}^{\text {backup }}$ fails after completing the phase 3. $u_{i}^{\text {backup }}$ is removed from its group and mobile agents can detect that $u_{i}^{\text {backup }}$ fails. 


\section{Conclusion}

This paper proposed a new strategy to improve scalability of mobile agent systems by applying an appropriate passive replication protocol to each replicated service according to its execution behavior, deterministic or non-deterministic. For this purpose, we presented the two passive replication protocols, PRPNS and $P R P D S$, for non-deterministic and deterministic services respectively. While ensuring linearizability [6], they both allow visiting mobile agents to be forwarded to and execute their tasks on any node performing a service agent, not necessarily the primary agent. Especially, the more lightweight protocol PRPDS allows any service agent to process each mobile agent request and coordinate with the other replica service agents after receiving the request and obtaining its delivery sequence number from the primary agent. Thus, if PRPDS is well-combined with existing load balancing schemes [2], the request processing and coordination load can be evenly distributed among a set of deterministic and replicated service agents based on the workload of each service agent.

\section{References}

1. P. Bellavista, A. Corradi and C. Stefanelli. The Ubiquitous Provisioning of Internet Services to Portable Devices. IEEE Pervasive Computing, Vol. 1, No. 3, pp. 81-87, 2002.

2. H. Bryhni, E. Klovning and O. Kure. A Comparison of Load Balancing Techniques for Scalable Web Servers. IEEE Network, 14:58-64, 2000.

3. N. Budhiraja, K. Marzullo, F. B. Schneider and S. Toueg. The primary-backup approach. Distributed Systems(S. Mullender ed., ch. 8, 199-216, Addison-Wesley, second ed., 1993.

4. X. Defago, A. Schiper and N. Sergent. Semi-Passive Replication. In Proc. of the 17th IEEE Symposium on Reliable Distributed Systems, pp. 43-50, 1998.

5. M. Fukuda, Y. Tanaka, N. Suzuki, L.F. Bic and S. Kobayashi. A Mobile-AgentBased PC Grid. In Proc. of the Fifth Annual International Workshop on Active Middleware Services, pp. 142-150, 2003.

6. M. Herlihy and J. Wing. Linearizability: a correctness condition for concurrent objects. ACM Transactions on Progr. Languages and Syst., 12(3):463-492, 1990.

7. K. Rothermel and M. Schwehm. Mobile Agents. In A.Kent and J.G.Williams(Eds.): Encyclopedia for Computer Science and Technology, 40(25):155$176,1999$.

8. M. Wiesmann, F. Pedone, A. Schiper, B. Kemme and G. Alonso. Understanding Replication in Databases and Distributed Systems. In Proc. of the 21st International Conference on Distributed Computing Systems, pp. 464-474, 2000. 